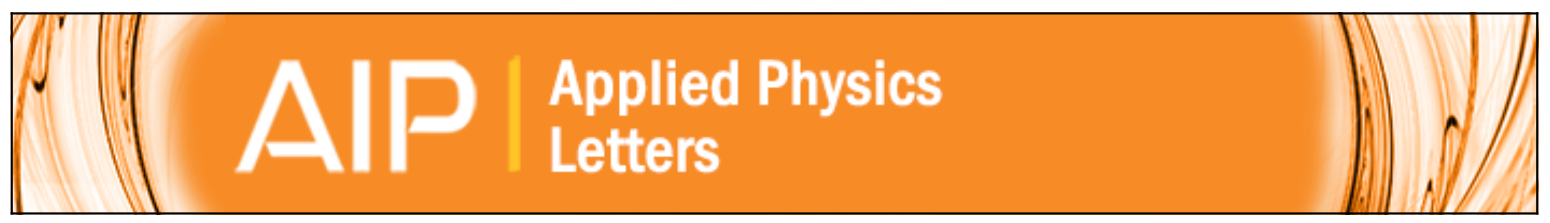

\title{
Microstructural imaging of high repetition rate ultrafast laser written $\mathrm{LiTaO} 3$ waveguides
}

Ben McMillen, Kevin P. Chen, and Daniel Jaque

Citation: Applied Physics Letters 94, 081106 (2009); doi: 10.1063/1.3088852

View online: $\mathrm{http}: / / \mathrm{dx}$. doi.org/10.1063/1.3088852

View Table of Contents: http://scitation.aip.org/content/aip/journal/apl/94/8?ver=pdfcov

Published by the AIP Publishing

\section{Articles you may be interested in}

$\mu$-Raman spectroscopy characterization of $\mathrm{LiNbO} 3$ femtosecond laser written waveguides

J. Appl. Phys. 112, 123108 (2012); 10.1063/1.4769869

Microspectroscopy of ultrafast laser inscribed channel waveguides in Yb:tungstate crystals

Appl. Phys. Lett. 98, 141108 (2011); 10.1063/1.3573999

Waveguiding and nonlinear optical properties of three-dimensional waveguides in $\mathrm{Li} \mathrm{Ta} O \mathrm{O}$ written by highrepetition rate ultrafast laser

Appl. Phys. Lett. 93, 111106 (2008); 10.1063/1.2980437

Raman gain from waveguides inscribed in $\mathrm{K} \mathrm{Gd}$ ( W O 4 ) 2 by high repetition rate femtosecond laser Appl. Phys. Lett. 92, 081105 (2008); 10.1063/1.2884188

Optical waveguide fabrication in z -cut lithium niobate ( $\mathrm{LiNbO} 3$ ) using femtosecond pulses in the low repetition rate regime

Appl. Phys. Lett. 88, 111109 (2006); 10.1063/1.2186389

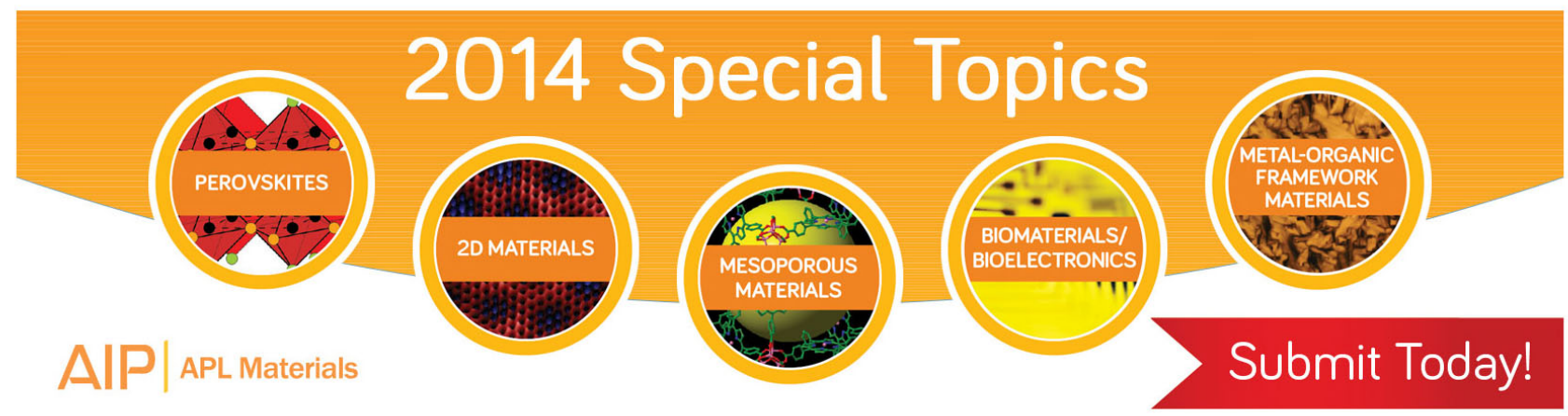




\title{
Microstructural imaging of high repetition rate ultrafast laser written $\mathrm{LiTaO}_{3}$ waveguides
}

\author{
Ben McMillen, ${ }^{1}$ Kevin P. Chen, ${ }^{1}$ and Daniel Jaque ${ }^{2, a)}$ \\ ${ }^{1}$ Departament of Electrical and Computer Engineering, University of Pittsburgh, Pittsburgh, \\ Pennsylvania 15261, USA \\ ${ }^{2}$ Departamento de Física de Materiales C-IV, Facultad de Ciencias, Universidad Autónoma de Madrid, \\ Madrid 28049, Spain
}

(Received 19 November 2008; accepted 4 February 2009; published online 24 February 2009)

\begin{abstract}
The microstructural changes associated with the formation of lithium tantalate waveguides after high repetition rate ultrafast laser inscription has been investigated by confocal micro-Raman experiments. While the laser beam focal volume is characterized by significant lattice damage, no reduction of Raman mode strength has been observed at the guiding region, suggesting the preservation of the nonlinear optical coefficient in the waveguide. A general blueshift of the Raman modes has been observed at the guiding region, suggesting lattice compression as the dominant mechanism of waveguide formation. (C) 2009 American Institute of Physics.
\end{abstract}

[DOI: 10.1063/1.3088852]

The ability of ultrafast laser pulses to precisely control the refractive index of transparent media at the micron scale is being extensively used for the fabrication of a great variety of three-dimensional photonic devices. ${ }^{1-4}$ Ultrafast direct laser writing (DLW) is becoming one of the most versatile and popular techniques for the fabrication of low loss optical channel waveguides in glasses, crystals, and transparent ceramics. ${ }^{1,5,6}$ Although low repetition rates have been widely used, ${ }^{6,7}$ the use of high repetition rates $(>250 \mathrm{kHz})$ has recently been found advantageous for the fabrication of low loss, highly symmetric waveguides. High repetition ultrafast waveguide inscription was first demonstrated in glasses ${ }^{1,8,9}$ and later in lithium niobate crystals. ${ }^{10,11}$ In the latter case, the use of high repetition rates has lead to the fabrication of waveguides with a minimal modification of the lithium niobate network and with the lowest propagation losses reported for a DLW lithium niobate waveguide $(0.6 \mathrm{~dB} / \mathrm{cm}){ }^{10,11}$

Very recently, high repetition rate $(250 \mathrm{kHz})$ ultrafast DLW of channel waveguides operating in the telecommunication range has also been demonstrated in lithium tantalate $\left(\mathrm{LiTaO}_{3}\right)$ crystals. $^{12}$ The obtained waveguides were fabricated without any degradation in the original nonlinear properties of the $\mathrm{LiTaO}_{3}$ network making them of special relevance for the future development of integrated nonlinear and optoelectronic integrated devices. In addition, it has been reported that the properties of the obtained waveguides (in terms of spatial extension, symmetry, and location) can be tailored by means of the writing conditions.

Despite the widespread interest in ultrafast DLW $\mathrm{LiTaO}_{3}$ waveguides, nothing is known about the photophysical and photochemical mechanisms responsible for the formation of these waveguides. The exact knowledge of these mechanisms is required for the understanding of the involved processes, being the first and essential step toward full control over the waveguide properties. Micro-Raman ( $\mu$-Raman) spectroscopy has been found to be a very sensitive tool for the detection of slight modifications in the $\mathrm{LiTaO}_{3}$ network, thus making possible the recognition of compositional

${ }^{\text {a)} E l e c t r o n i c ~ m a i l: ~ d a n i e l . j a q u e @ u a m . e s . ~}$ changes, lattice disorder, irreversible lattice damage, and changes in the interionic distances, all with a submicron spatial resolution. ${ }^{12-14}$

In this work, we report on confocal Raman imaging of high repetition rate ultrafast DLW $\mathrm{LiTaO}_{3}$ optical waveguides obtained under different writing conditions. The Raman images have been compared to optical images of the waveguide cross section as well as propagation mode images so that the possible mechanisms leading to waveguide formation may be discussed and identified.

The waveguides studied in this work were fabricated with a Coherent MIRA oscillator and a RegA regenerative amplifier system producing 150 fs pulses at a wavelength of $770 \mathrm{~nm}$ and a repetition rate of $250 \mathrm{kHz}$. The writing beam was focused into a $Z$-cut $\mathrm{LiTaO}_{3}$ sample at a depth of $150 \mu \mathrm{m}$ with a $40 \times$ aspheric lens (numerical aperture =0.68). The $\mathrm{LiTaO}_{3}$ sample was mounted on a three-axis motorized stage and translated at speed of $1 \mathrm{~mm} / \mathrm{s}$ allowing inscription of waveguides parallel to the $x$ crystallographic axis. Pulse energy was varied from $100 \mathrm{~nJ}$ up to $1.6 \mu \mathrm{J}$. Further details of the waveguide fabrication procedure can be found elsewhere. ${ }^{12}$

Figure 1(a) shows a transmission image of the cross section of a waveguide written with a pulse energy of $300 \mathrm{~nJ}$. Laser pulses were incident from the top of this image. A laser modification track $(\approx 20 \mu \mathrm{m}$ long and $\approx 2 \mu \mathrm{m}$ width $)$ is clearly denoted by a reduction in the transmitted intensity. Figure 1(b) shows the corresponding $1.55 \mu \mathrm{m}$ guiding mode indicating the formation of a highly symmetric waveguide just above the laser modified region. $\mu$-Raman investigations were performed by using a Horiba Jobin Yvon fiber coupled confocal microscope. The $488 \mathrm{~nm}$ excitation beam was focused into the waveguide's cross section by using an oil immersion $100 \times$ UplanSApo (numerical aperture=1.4) microscope objective. The spatial and spectral resolutions of the confocal system were estimated to be $200 \mathrm{~nm}$ and $0.4 \mathrm{~cm}^{-1}$, respectively. The $\mathrm{LiTaO}_{3}$ sample was positioned on a two-axis stage allowing for a spatial Raman spectral map of the waveguide's cross section. Figure 1(c) shows a typical $x(z z) \bar{x} \mu$-Raman spectrum as obtained from an un- 

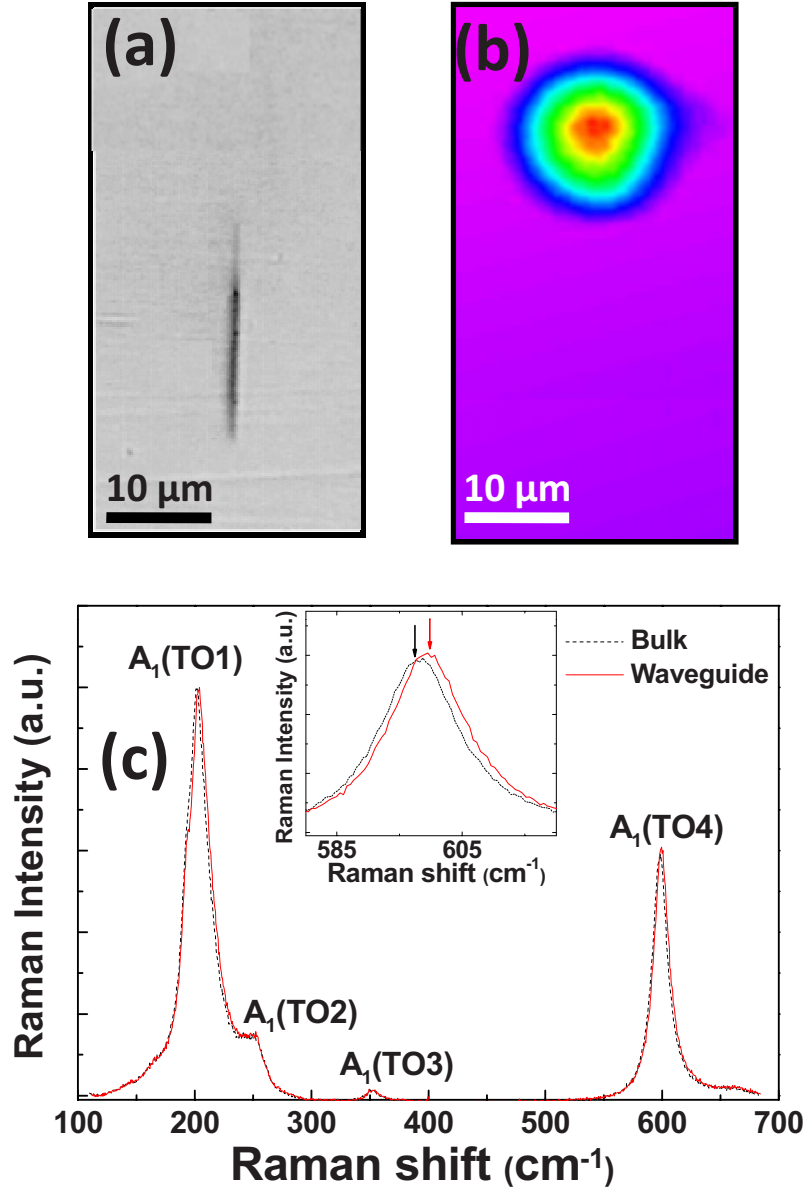

FIG. 1. (Color online) (a) Optical transmission micrograph of the laser modification track written with a pulse energy of $300 \mathrm{~nJ}$ at a translation speed of $1 \mathrm{~mm} / \mathrm{s}$. (b) Near field mode profile from the adjacent guiding region at $1550 \mathrm{~nm}$ wavelength. (c) $x(z z) \bar{x} \mu$-Raman spectrum obtained from the bulk and waveguide. Each Raman mode is properly labeled. Inset shows a detail of the $A_{1}\left(\mathrm{TO}_{4}\right)$ Raman mode denoting the blueshift generated at the waveguide.

treated $\mathrm{LiTaO}_{3}$ region (bulk). The different $A(\mathrm{TO})$ Raman modes have been properly identified and labeled according to prior works. ${ }^{13}$ For the sake of comparison, the $\mu$-Raman spectrum obtained at the waveguide's location has been also included in Fig. 1(c). Both spectra look very similar in terms of overall intensity and number of active Raman modes, suggesting the absence of relevant lattice damage (which would lead to a decrease in the Raman intensity) as well as to a nonrelevant crystal reorientation at the waveguide's location (which would lead to the appearance of Raman modes forbidden in this configuration). Nevertheless, a detailed investigation of both Raman spectra denotes a slight shift of the Raman modes. As an example, inset in Fig. 1(c) shows a clear shift toward larger vibration energies in the $A_{1}(\mathrm{TO} 4)$ Raman mode. Similar shifts have also been found for the $A_{1}$ (TO1) and $A_{1}$ (TO2) Raman modes. In the case of the $A_{1}$ (TO3) mode, due to its weak intensity, the error associated to the fitting procedure did not allow us to determine any possible shift. According to previous works, ${ }^{15}$ the almost general blueshift of the Raman modes suggests the presence of a local compressive stress in the guiding region, as has been recently reported in ultrafast DLW waveguides fabricated in potassium gadolinium tungstate crystals. ${ }^{16}$ According to the Clausius Mossotti relation, ${ }^{17}$ this compressive stress would account for an increase in the local refractive

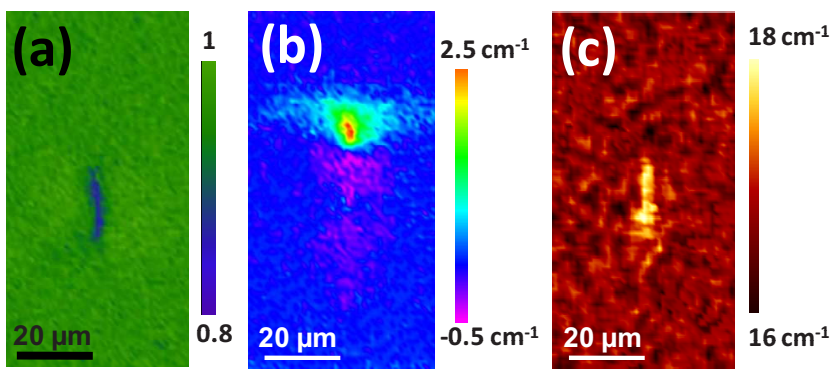

FIG. 2. (Color online) Spatial map of the integrated Raman intensity (a), of the induced shift and line-width of the $A_{1}\left(\mathrm{TO}_{4}\right)$ Raman mode [(b) and (c), respectively]. Data correspond to the waveguide written with a pulse energy of $300 \mathrm{~nJ}$ at a translation speed of $1 \mathrm{~mm} / \mathrm{s}$.

index and hence for the formation of the waveguide provided the fact that the pressure induced change in the lattice density is dominant over the pressure induced changes in the average electronic polarizability. ${ }^{18}$ In order to correlate these changes with the waveguide formation, we have recorded Raman images of the waveguide's cross section. Results for the integrated Raman intensity, Raman shift, and width of the $A_{1}$ (TO4) Raman mode are plotted in Figs. 2(a)-2(c), respectively. Similar images have been obtained from the analysis of the $A_{1}(\mathrm{TO} 1)$ and $A_{1}(\mathrm{TO} 2)$ Raman modes. Nevertheless, the best images (in terms of contrast and signal-to-noise ratio) were obtained from the analysis of the $A_{1}(\mathrm{TO} 4)$ mode since it is well isolated from the other Raman modes, minimizing the error in the fitting procedure. Figure 2(a) shows a $20 \%$ reduction of the overall Raman intensity in the laser modification track, indicating the formation of a damaged volume characterized by a large density of extended defects. This conclusion is further supported by the broadening of Raman modes at the focal volume [depicted in Fig. 2(c)] denoting a local disorder. It is also in agreement with previous works indicating a remarkable deterioration in the nonlinear coefficients of the $\mathrm{LiTaO}_{3}$ lattice at the focal volume. ${ }^{12}$ Figure 2(b) denotes that the induced shift in the Raman modes has an almost circular shape, and it is located a few microns above the laser damage track, in accordance with the waveguide location extracted from Fig. 1(b). From Figs. 2(a) and 2(b), it is clear that the compressive stress (determining the extension and position of the waveguide) has a negligible spatial overlap with the damaged volume. This fact is of special relevance since it ensures minimum propagation losses (waveguide mode is not propagating through a damaged volume).

Raman measurements shown in Fig. 2(b) provide telltale information about waveguide formed by the ultrafast laser writing process. First, there is no noticeable reduction of the integrated Raman signal in the waveguiding region shown in Fig. 2(a). This indicates the preservation of $\mathrm{LiTaO}_{3}$ crystalline structures and its nonlinear optical properties, a necessity for any waveguide fabrication approach used for electrooptical device fabrications. Second the full width at half maximum (FWHM) of the laser-induced Raman mode shift shown in Fig. 2(b) provides better estimation of the size of the waveguide. A Gaussian fitting yields FWHMs of 6.2 and $7.1 \mu \mathrm{m}$ along vertical and horizontal directions, respectively, for the induced Raman mode shift in the waveguiding region. This is in contrast to the $<2 \mu \mathrm{m}$ lateral size of laser damage track obtained by the optical microscope visual inspection Third, the magnitude of the maximum induced shift 

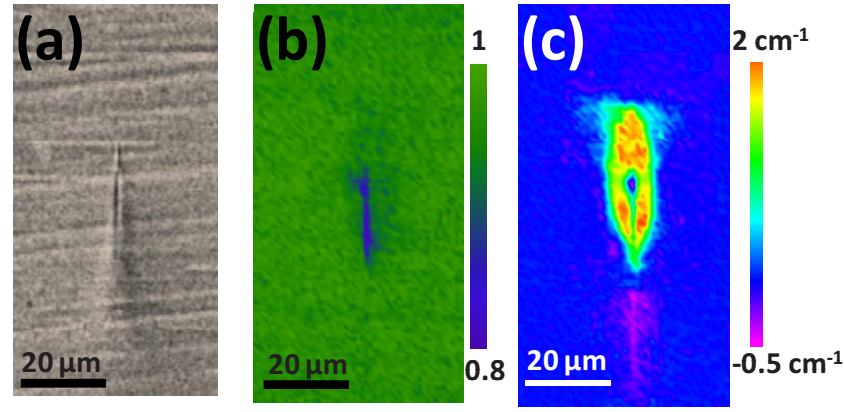

FIG. 3. (Color online) (a) Optical transmission micrograph of the laser modification track written with a pulse energy of $1 \mu \mathrm{J}$ at a translation speed of $1 \mathrm{~mm} / \mathrm{s}$. Spatial map of the integrated Raman intensity and of the induced shift in the $A_{1}\left(\mathrm{TO}_{4}\right)$ Raman mode [(b) and (c), respectively].

in the Raman mode shown in Fig. 2(b) can be also used to get a rough estimation of the compression induced. Based on the Grüneisen parameter of the $A_{1}(\mathrm{TO} 4)$ Raman mode $\left(0.2 \mathrm{~cm}^{-1} / \mathrm{kBar}\right),{ }^{15}$ a maximum compressive residual stress of $\approx 10 \mathrm{kBar}$ is estimated at waveguide's location. The fact that this local compression caused refractive index increment suggests that the induced change in the $\mathrm{LiTaO}_{3}$ unit cell volume (estimated to be $\Delta V / V \approx-10^{-2}$ from the averaged Youngs modulus of $\left.\mathrm{LiTaO}_{3}\right)^{19}$ is dominant over the possible pressure induced reduction in the electronic polarizability.

We have also obtained the Raman images of a waveguide written with higher pulse energies. Figure 3(a) shows an optical transmission image of the cross section of a waveguide written with a pulse energy of $1 \mu \mathrm{J}$. For this case, butt-coupling experiments have revealed that the location of the guiding mode is strongly dependent on the launching conditions. In general, we have found that guiding is possible above, below, and at both laterals from the laser modified zone, which is in contrast with the well localized waveguiding found in the previous structure written at $300 \mathrm{~nJ}$ [Fig. 1(b)]. The focal volume is characterized by a reduction in the integrated Raman intensity, which is again related to lattice damage at the focal volume [see Fig. 3(b)]. In the surroundings of this damaged core, a blueshift of the Raman modes is observed, which can be seen in Fig. 3(c). Following the same argument as in the case of the low-energy waveguide, Fig. 3 (c) suggests that when the pulse energy is increased, the femtosecond laser interaction generates local lattice compression (refractive index increase) surrounding the focal volume. This compression is accompanied by a slight dilatation (refractive index decrease) generated within the focal volume as well as below it, which is denoted by redshift generated along the irradiation direction. If lattice compression/dilatation is again related to a refractive index increase/decrease, the refractive index map suggested by Fig. 3 is quite similar to that previously reported for high repetition rate ultrafast DLW waveguides in chalcogenide glasses and well explains the possible light coupling at different spatial locations in the surroundings of the focal volume. ${ }^{20}$

Finally, it should be mentioned that the Raman images of Fig. 2 are completely different than those recently obtained for high repetition rate ultrafast $\mathrm{DLW} \mathrm{LiNbO}_{3}$ waveguides in which the waveguide's volume was characterized by a redshift of Raman modes. ${ }^{21}$ The different Raman images denote the different mechanisms involved in the formation of the waveguide. In the case of high repetition rate $\mathrm{LiNbO}_{3}$ laser writing, waveguide formation was postulated to be created as a consequence of a change in the spontaneous polarization brought on by a slight ionic rearrangement, whereas in the case of $\mathrm{LiTaO}_{3}$ this work concludes that lattice compression is the main mechanism causing the local increase in the refractive index.

In summary, we report on the microstructural lattice changes associated with high repetition rate ultrafast laser written $\mathrm{LiTaO}_{3}$ waveguides. We have found that femtosecond laser interaction leads to a relevant lattice damage at the focal volume accompanied by a local lattice compression in its surroundings, which we believe is the origin of the waveguide formation (i.e., of the local refractive index increase). The location and extent of the induced lattice compression have been found to be strongly dependent on the writing energy and have been used to explain the differences between the guiding properties of $\mathrm{LiTaO}_{3}$ waveguides fabricated with low and high pulse energies.

This work has been supported by the Universidad Autónoma de Madrid ad Comunidad Autonoma de Madrid (Project No. CCG07-UAM/MAT-1861), by the Spanish Ministerio de Educacion y Ciencia (Grant No. MAT 200764686), and by the National Science Foundation of the U.S. (Grant No. 0556086)

${ }^{1}$ K. M. Davis, K. Miura, N. Sugimoto, and K. Hirao, Opt. Lett. 21, 1729 (1996).

${ }^{2}$ G. Zhou and M. Gu, Opt. Lett. 31, 2783 (2006)

${ }^{3}$ N. Takeshima, Y. Narita, S. Tanaka, Y. Kuroiwa, and K. Hirao, Opt. Lett. 30, 352 (2005).

${ }^{4}$ Y. Liao, J. Xu, Y. Cheng, Z. Zhou, F. He, H. Sun, J. Song, X. Wang, Z. Xu, K. Sugioka, and K. Midorikawa, Opt. Lett. 33, 2281 (2008).

${ }^{5}$ S. Nolte, M. Will, J. Burghoff, and A. Tunnermann, Appl. Phys. A: Mater. Sci. Process. 77, 109 (2003).

${ }^{6}$ G. Torchia, A. Ródenas, A. Benayas, E. Cantelar, L. Roso, and D. Jaque, Appl. Phys. Lett. 92, 111103 (2008).

${ }^{7}$ R. R. Thomson, S. Campbell, I. J. Blewett, A. K. Kar, and D. T. Reid, Appl. Phys. Lett. 88, 111109 (2006).

${ }^{8}$ R. Osellame, N. Chiodo, V. Maselli, A. Yin, M. Zavelani-Rossi, G. Cerullo, P. Laporta, L. Aiello, S. De Nicola, P. Ferraro, A. Finizio, and G. Pierattini, Opt. Express 13, 612 (2005).

${ }^{9}$ S. M. Eaton, H. Zhang, P. R. Herman, F. Yoshino, L. Shah, J. Bovatsek, and A. Y. Arai, Opt. Express 13, 4708 (2005).

${ }^{10}$ A. H. Nejadmalayeri and P. R. Herman, Opt. Express 15, 10842 (2007).

${ }^{11}$ R. Osellame, M. Lobino, N. Chiodo, M. Marangoni, G. Cerullo, R. Ramponi, H. T. Bookey, R. R. Thomson, N .D. Psaila, and A. K. Kar, Appl. Phys. Lett. 90, 241107 (2007).

${ }^{12}$ B. McMillen, K. P. Chen, H. An, S. Fleming, V. Hartwell, and D. Snoke, Appl. Phys. Lett. 93, 111106 (2008).

${ }^{13}$ Y. Repelin, E. Husson, F. Bennani, and C. Proust, J. Phys. Chem. Solids 60, 819 (1999).

${ }^{14}$ L. Shi, Y. Kong, W. Yan, J. Sun, S. Chen, L. Zhang, W. Zhang, H. Liu, X. Li, X. Xie, D. Zhao, L. Sun, Z. Wang, J. Xu, and G. Zhang, Mater. Chem. Phys. 95, 229 (2006)

${ }^{15}$ A. Jayaraman and A. A. Ballman, J. Appl. Phys. 60, 1208 (1986).

${ }^{16}$ S. M. Eaton, C. A. Merchant, R. Iyer, A. J. Zilkie, A. S. Helmy, J. S. Aitchison, P. R. Herman, D. Kraemer, R. J. D. Miller, C. Hnatovsky, and R. S. Taylor, Appl. Phys. Lett. 92, 081105 (2008).

${ }^{17}$ C. Kittel, Introduction to Solid State Physics, 8th ed. (Wiley, New York, 2005).

${ }^{18}$ A. Benayas, D. Jaque, S. J. Hettrick, J. S. Wilkinson, and D. P. Shepherd, J. Appl. Phys. 103, 103104 (2008).

${ }^{19} \mathrm{http} / / / \mathrm{www}$. korth.de.

${ }^{20}$ M. Hughes, W. Yang, and D. Hewak, Appl. Phys. Lett. 90, 131113 (2007).

${ }^{21}$ A. Ródenas, A. H. Nejadmalayeri, D. Jaque, and P. R. Herman, Opt. Express 16, 13979 (2008). 